Casos Clínicos

Arch. Esp. Urol., 61, 5 (637-640), 2008

\section{CARCINOMA DUCTAL DEL UTRÍCULO PROSTÁTICO. CASO CLÍNICO Y REVISIÓN DE LA LITERATURA}

\author{
Nerea Senarriaga Ruiz de la Illa, Ana Loizaga \\ Iriarte, José Ramón Larrinaga Izaguirre, Isabel Lacasa \\ Viscasillas, Jesús María Arciniega García y Miguel \\ Unda Urzaiz.
}

Servicio de Urología. Hospital de Basurto. Bilbao. España.

Resumen.- OBJETIVO: Se presenta un caso de adenocarcinoma ductal prostático, anteriormente conocido como carcinoma endometrioide y se revisa la literatura.

MÉTODOS: Paciente de 75 años que acude a la consulta por sintomatología de obstrucción urinaria, PSA de 8,1 $\mathrm{ng} / \mathrm{ml}$., sin otra clínica acompañante. El tacto rectal y la ecografía abdominopélvica evidencian una próstata pequeña y pétrea. Se realiza resección transuretral desobstructiva y la anatomía patológica revela carcinoma ductal del utrículo prostático.

RESULTADOS: A los 9 años del diagnóstico no presenta signos de diseminación.

Nerea Senarriaga Ruiz de la llla

Servicio de Urología

Hospital de Basurto

Bilbao. (España).

netxu_22@yahoo.es

Trabajo recibido: 1 de octubre 2007.
CONCLUSIONES: De hallazgo casual en el estudio anatomo-patológico, el adenocarcinoma ductal del utrículo prostático es una rara neoformación, cuya incidencia entre todos los carcinomas prostáticos ha sido citada entre $0,2-0,8 \%$, si bien resulta interesante hacer una revisión sobre el tema para fomentar su estudio. Se revisan la clínica, diagnóstico y tratamiento de dicho carcinoma.

Palabras clave: Obstrucción urinaria. Utrículo prostático. Carcinoma ductal. Próstata.

Summary.- OBJECTIVE: A case of ductal carcinoma of the prostatic utricle is described, previously known as endometrial carcinoma, and literature is reviewed.

METHODS: 75 years old patient who consults for lower urinary tract obstructive symptoms, with a PSA of $8.1 \mathrm{ng} / \mathrm{ml}$., without more symptoms. Digital rectal examination and ultrasound showed a small and stony prostate. Deobstructive transurethral resection of the prostate was performed and the biopsy revealed ductal carcinoma of the utricle.

RESULTS: Nine years after diagnosis signs of dissemination are not present.

CONCLUSIONS: As incidental finding in the biopsy, ductal carcinoma of the utricle is a rare tumor, the incidence of which among all prostatic carcinomas has been cited as $0.2-0.8 \%$. We report a review about this topic for improving its knowledge. Symptoms, pathological findings and treatment of this carcinoma have been reviewed.

Keywords: Urinary obstruction. Utricle. Ductal carcinoma. Prostate.

\section{INTRODUCCIÓN}

El carcinoma ductal tiene lugar en la uretra prostática y en los conductos prostáticos periuretrales. Anteriormente fue denominado carcinoma endometrioide, no sólo porque presenta una similitud histológica con el adenocarcinoma endometrial de útero, sino porque se consideró que provenía de los restos müllerianos del utrículo prostático. Por ello se pensó que estos tumores podían ser estrogenodependientes y como consecuencia de ello la terapia hormonal (estrogénica) y la orquiectomía estarían contraindicadas. Sin embargo, hasta el momento no hay evidencia de que estos tumores deriven del epitelio mülleriano. Recientemente, se ha demostrado que el carcinoma ductal es una variante inusual del adenocarcinoma prostático, de acuerdo con la evidencia clínica, embrionaria, enzimática, morfológica e inmunológica $(1,2)$.

La singularidad de esta patología, su bajo índice de sospecha en la práctica clínica diaria debido a su infre- 
cuente aparición y la escasa referencia a este tipo tumoral estadísticamente, nos ha animado a su presentación mediante un caso clínico.

\section{CASO CLÍNICO}

Paciente de 75 años de edad que acude a nuestras consultas por referir incontinencia urinaria y flujo miccional disminuido desde hace 3 meses.

Entre sus antecedentes personales destaca arritmia cardíaca e hipertensión.

En la exploración se observa globo vesical y al tacto rectal se objetiva una próstata plana y pétrea.

Se coloca sonda uretral evidenciándose un residuo de $400 \mathrm{cc}$.

La analítica de sangre es normal, excepto creatinina de $1,63 \mathrm{mg} / \mathrm{dl}$ y PSA de $8,1 \mathrm{ng} / \mathrm{ml}$.

Ecografía abdominopélvica: Próstata pequeña con imagen vesical de dudoso proceso neoformativo (Figura 1).

Se le realiza resección transuretral de próstata descartándose tumor vesical en la misma intervención. El diagnóstico anatomopatológico fue de carcinoma ductal de utrículo prostático (Figura 2).

Debido a elevaciones progresivas del PSA, se instaura tratamiento con bloqueo androgénico máximo a los 3 años de la cirugía.

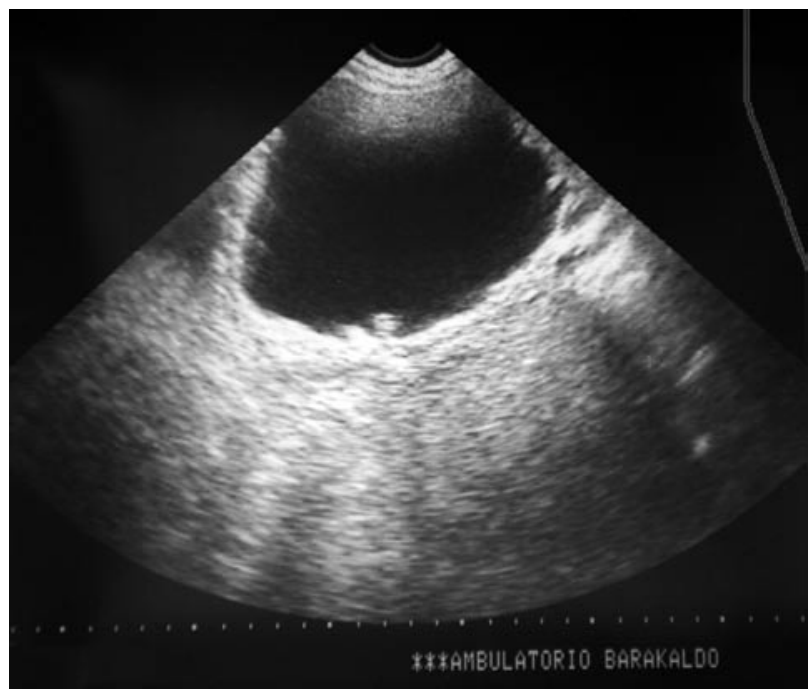

FIGURA 1. Imágen ecográfica: dudoso proceso neoformativo en vejiga.
El último control fue 9 años después de la RTU de próstata presentando un PSA de $41 \mathrm{ng} / \mathrm{ml}$ y sin datos objetivos de metástasis (Figura 3).

\section{DISCUSIÓN}

El adenocarcinoma ductal o endometrioide es una entidad muy infrecuente, de ahí la escasez de casos hallados en la revisión bibliográfica realizada. Fue descrita por primera vez por Melicow y Pachitter en 1967 (3).

Tiene lugar exclusivamente en hombres de edad avanzada, los cuales refieren síntomas indistinguibles de cualquier otra patología prostática.

Endoscópicamente, se pueden observar multitud de masas blanquecinas, polipoides, exofíticas, de aspecto papilar y friables, que sangran fácilmente al contacto con el cistoscopio y protruyen desde los ductos hacia el utrículo prostático en el ápex del verumontanum, con - sin infiltración de éste. La glándula prostática puede llegar a mostrar en muchas ocasiones una nodularidad palpable.

En el momento de la presentación, la mayoría de los tumores están confinados a la próstata $(1,3)$ con unos niveles séricos del PSA a menudo normales, salvo en pacientes con estadíos avanzados (4). Esta discrepancia en los niveles séricos del antígeno prostático específico podría explicarse por el comienzo temprano de la sintomatología obstructiva, lo que lleva al paciente a consultar y ser intervenido por su clínica miccional, consiguiendo por tanto un rápido diagnóstico del car-

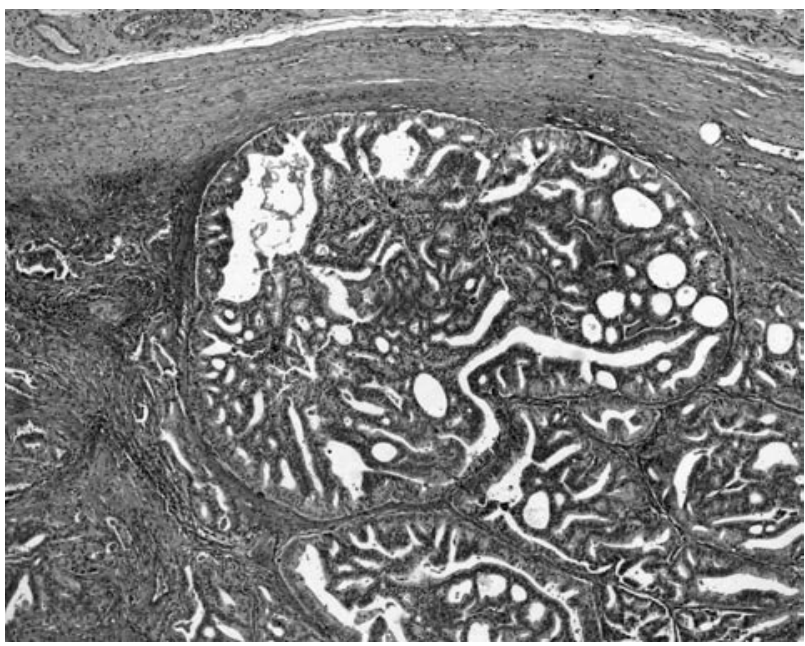

FIGURA 2. Hallazgos histopatológicos. 
cinoma ductal centralmente localizado (periuretral o intrauretral), en comparación con el carcinoma prostático periférico. Este rápido diagnóstico hace que en muchos casos, el carcinoma ductal no se haya desarrollado excesivamente y por tanto el volumen tumoral sea pequeño.

El adenocarcinoma prostático con rasgos endometrioides habitualmente implica a los grandes conductos prostáticos periuretrales y al verumontanum (1). Microscópicamente, se trata de una proliferación neoplásica de glándulas, con áreas papilares tapizadas por un epitelio columnar pseudoestratificado atíicico que lo hace, al menos focalmente indistinguible del adenocarcinoma uterino (4). Se encuentra bien delimitado y está compuesto por papilas más o menos anastomosadas, con células cúbicas y basófilas que expresan PAP y PSA, idénticas a las que recubren el utrículo del adulto. El probable origen sea el revestimiento postpuberal por células prostáticas y por tanto no existe justificación alguna para considerar a este tumor de origen mülleriano, hormonorresistente desde su inicio, aunque se cree que se trata de una variedad con un alto grado de agresividad (5) (Figura 3).

Dos patrones de crecimiento han sido observados: papilas intraductales y glándulas ramificadas. Estos dos patrones coexisten en aproximadamente la mitad de los casos y ambos muestran a menudo anaplasia nuclear y frecuentes mitosis. El carcinoma ductal tiene como ca-

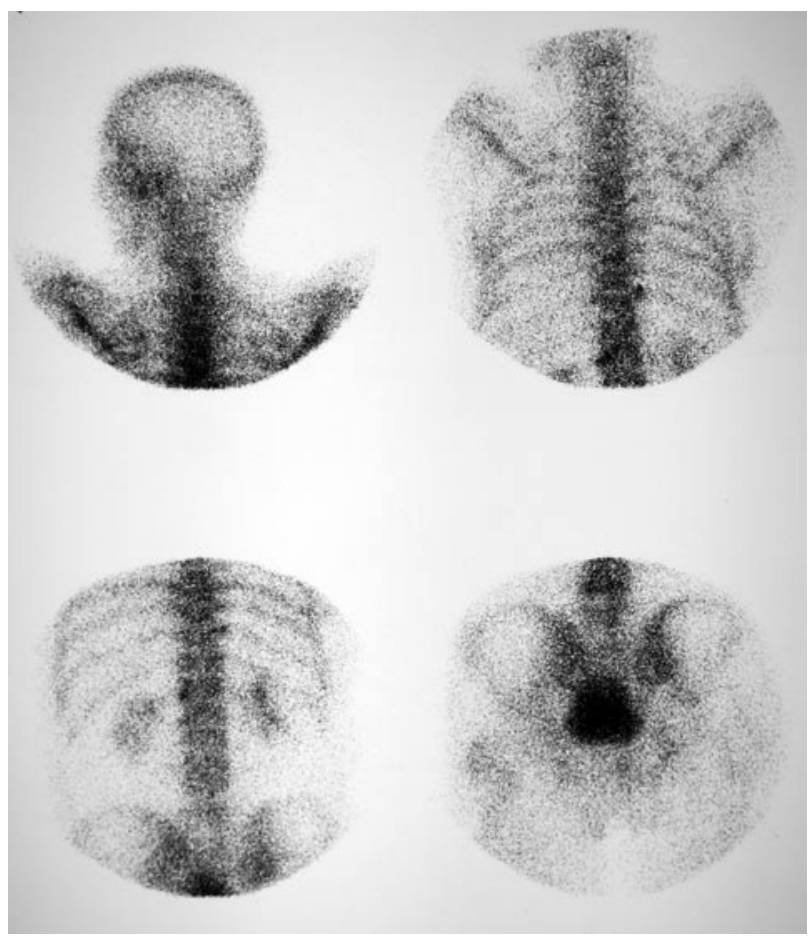

FIGURA 3. Gammagrafía ósea. racterística un crecimiento exofítico dentro de espacios luminares, tales como grandes conductos o la uretra. El limitado espacio que existe en la región periférica de la próstata no permite una correcta formación de papilas o complejos glandulares ramificados, lo cual apoya el hecho de la rareza o ausencia de tumores con patrón endometrioides en esa localización.

El adenocarcinoma con rasgos endometrioides invariablemente expresa una intensa inmunorreactividad citoplásmica para PAP y para PSA $(1,6)$. El desarrollo de técnicas de inmunoperoxidasa y la habilidad de localización de tejidos con antígeno específico hablan en favor de un origen prostático en el carcinoma endometrial del utrículo prostático (3).

En cuanto al tratamiento y pronóstico decir que, aunque nosotros hemos tenido la oportunidad de tratar un caso con evolución favorable, ya que nuestro paciente a los 9 años del diagnóstico no presentaba metástasis en los estudios de imagen, el carcinoma ductal de próstata es más agresivo de lo que previamente se pensaba, alcanzando una supervivencia a 5 años de entre el 15 y el $43 \%$. El patrón de metástasis es idéntico al del adenocarcinoma prostático acinar, siendo el hueso, cerebro, pulmones y nódulos linfáticos, los sitios más comunes de presentación. El distintivo de patrón endometrioide es altamente predictivo de carcinoma invasivo y de metástasis subsecuentes (7).

Una terapia adyuvante proporciona alivio paliativo en muchos de los casos, pero no parece influir en cuanto a la supervivencia se refiere. La radioterapia ha sido utilizada con el fin de paliar la hematuria o la dificultad de vaciamiento, así como control del dolor óseo si existieran metástasis. La experiencia sugiere que estos tumores son sensibles al tratamiento con radiación.

Estudios recientes afirman que este tumor es correctamente tratado de manera similar al adenocarcinoma típico. Sin embargo, su pronóstico es notablemente peor.

En cuanto al diagnóstico diferencial, el carcinoma ductal debe ser diferenciado del carcinoma prostático de células transicionales, tejido prostático ectópico, pólipos benignos, adenoma nefrogénico, uretritis papilar proliferativa y papiloma invertido (1).

\section{CONCLUSIÓN}

El adenocarcinoma ductal prostático fue reconocido como una variante inusual de carcinoma prostático en 1967 por Melicow. Su histogénesis, comportamiento biológico y tratamiento han sido debatidos y existen opiniones enfrentadas en diferentes series.

Por otra parte, resulta extraña la falta de comunicaciones en la revisión bibliográfica, lo cual no ayuda demasiado a su correcto conocimiento y manejo. 


\section{BIBLIOGRAFIA y LECTURAS RECOMENDADAS (*lectura de interés $y^{* *}$ lectura fundamental)}

**1. SHAJU, L.; VARGHESE, D.N.B.; GARY, D. y cols.: "The prostatic gland: Malignancies other than adenocarcinomas". California, 38: 180, 2000.

2. GUY, L.; BEGIN, L.R.; AL-OTHMAN, K. y cols.: "Neuroendocrine cells of the verumontanum: A comparative inmunohistochemical study". J. Urol., 82: 738, 1998.

*3. CHESA, N.; DEL ROSARIO, J.; NUÑEZ, J. y cols.: "Carcinoma endometriode del utrículo prostático". Arch. Esp. Urol., 40: 281, 1987.

4. JIMÉNEZ CRUZ, J.F.; RIOJA SANZ, L.A.: "Tratado de Urología". 1965. Prous Science. Tomo IV, Barcelona. 2006

*5. ALGABA, F.: “Atlas de patología de los tumores urogenitales. Diagnóstico morfológico y correlación clínico-patologica". 224-225. Pulso ediciones.

6. LEE, S.S.: "Endometrioid adenocarcinoma of the prostate: A clinicopathologic and inmunohistochemical study". Journal of Surgical Oncology, 55: 235, 1994.

7. LLARENA IBARGUREN, R.; LECUMBERRI CASTAÑOS, D.; PADILLA NIEVA, J. y cols.: "Carcinoma ductal prostático”. Arch. Esp. Urol., 56: 1054, 2003.

\section{Casos Clínicos}

Arch. Esp. Urol., 61, 5 (640-643), 2008

\section{LITIASIS VESICAL SECUNDARIA A MIGRACIÓN DE DISPOSITIVO INTRAUTERINO. REPORTE DE UN CASO}

\author{
Tomás Lázaro Rodríguez Collar, Yamel Gil del \\ Valle, Basily Valdés Estévez, Víctor Osvaldo Barquín \\ Carmona y José Antonio García Monzón.
}

Servicio de Urología. Hospital Universitario Dr. Carlos J. Finlay. La Habana. Cuba.

Resumen.- OBJETIVO: Presentar un caso clínico de litiasis vesical secundaria a la migración de un dispositivo intrauterino, su sintomatología, los medios empleados para su diagnóstico y el tratamiento aplicado.

MÉTODO/RESULTADO. Paciente de 30 años de edad, que al cabo del año de colocársele un dispositivo intrauterino (T de cobre), luego de interrupción de embarazo mediante legrado endometrial, comenzó a presentar síntomas urinarios irritativos bajos. Se le trató durante dos años como si tuviese cistitis infecciosa con varios ciclos de diferentes antibióticos. Por la persistencia de las molestias asiste a la consulta de Urología, y se le detecta, mediante ultrasonido y radiografía ántero-posterior de la pelvis, una litiasis intravesical de cinco por tres centímetros de tamaño, con una $T$ de cobre en su interior. Se le realizó la cistolitotomía suprapúbica, presentando una buena evolución y regresión total de los síntomas.

CONCLUSIÓN. La migración de un dispositivo intrauterino a la vejiga, es una causa infrecuente de litiasis vesi-

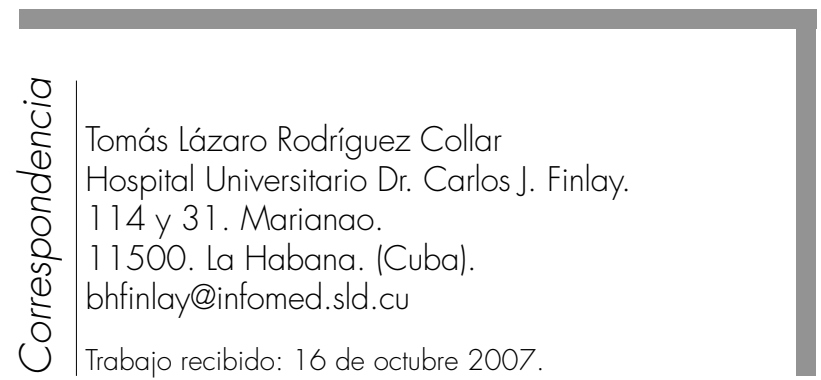

